\title{
Mariusz Szczygieł au pays des Tchèques ou les confessions d'un Polonais neurasthénique
}

Mariusz Szczygiet in Czechland. Or the Confessions of a Neurasthenic Pole

Marie-Odile Thirouin

\section{OpenEdition}

\section{Journals}

Édition électronique

URL : https://journals.openedition.org/recherchestravaux/854

DOI : 10.4000/recherchestravaux.854

ISSN : 1969-6434

Éditeur

UGA Éditions/Université Grenoble Alpes

Édition imprimée

Date de publication : 1 décembre 2016

Pagination : 67-82

ISBN : 978-2-84310-338-4

ISSN : 0151-1874

Référence électronique

Marie-Odile Thirouin, « Mariusz Szczygieł au pays des Tchèques ou les confessions d'un Polonais neurasthénique », Recherches \& Travaux [En ligne], 89 | 2016, mis en ligne le 12 janvier 2017, consulté le 29 octobre 2021. URL : http://journals.openedition.org/recherchestravaux/854 ; DOI : https:// doi.org/10.4000/recherchestravaux.854 


\section{Mariusz Szczygieł au pays des Tchèques ou les confessions d'un Polonais neurasthénique}

«Mes livres sur la Tchéquie sont destinés à démoraliser les Polonais...» : cette déclaration de Mariusz Szczygieł, extraite d'un entretien avec le journaliste tchèque Jiř́ Lojín pour le magazine littéraire en ligne Vaše Literatura, date de décembre $2012^{1}$. C'est une phrase instructive, en dépit de son caractère provocateur qui pourrait la faire négliger, car elle désigne à la fois un lectorat ou plutôt une cible, les compatriotes polonais de Mariusz Szczygiel, et une intention agressive : en écrivant sur la Tchéquie, celui-ci se livre à une entreprise de déstabilisation de ses lecteurs privilégiés. Pourquoi tant de haine, demandera-t-on? Il répondrait peut-être qu'il s'agit moins de haine que d'un amour paradoxal pour les siens ${ }^{2}$, à la manière de celui que manifeste un Thomas Bernhard pour l'Autriche, d'autant que le dernier livre de Szczygieł sur les Tchèques s'achève par le constat humiliant pour l'écrivain qu'en définitive, et malgré qu'il en ait, il ressemble comme deux gouttes d'eau à ces Polonais dont il voudrait ne pas être : «le Polonais en moi s'est relevé d'entre les morts», écrit-il, sarcastique,

I. M. Szczygieł, «Nepíšu speciálně pro Čechy» [Je n’écris pas en particulier pour les Tchèques], p. I, entretien en polonais du I6 décembre 2012 avec J. Lojín qui en donne la traduction tchèque, accompagnée de la version originale polonaise intitulée précisément "Moje ksią̇ki o Czechach mają demoralizować Polaków [...]", disponible sur <http://www.vaseliteratura.cz/rozhovory/ 47-clanky/2686-rozhovor-marius-szczygiel.html/> : « Mé knihy o Česku slouží k tomu, aby demoralizovaly Poláky [...]».

2. M. Szczygieł, Chacun son paradis, trad. M. Carlier, Arles, Actes Sud, 20I2, p. 206 : «Il se trouve par ailleurs que cet afflux de témoignages de sympathie m'a été adressé à moi, un être perpétuellement mécontent de son propre pays, bien que je considère que ce mécontentement exprime au fond une forme d'amour.» 
tournant en dérision à la fois ses propres proclamations d'athéisme et le dédain qu'il avait affecté jusque-là de la polonités.

On l'a compris : la provocation et l'autodérision définissent l'ethos du reporter Szczygieł, brouillant l'intention profonde de son étude du caractère et de la culture tchèques. J'admettrai toutefois que la «mission de démoralisation vis-à-vis des Polonais ${ }^{4}$ ", dont il se charge lui-même dans l'entretien avec Jiř́ Lojín, est bien le ressort d'un intérêt pour les Tchèques qui n'est que «second» : le pays des Tchèques, découvert par Szczygieł il y a une quinzaine d'années, vaut à ses yeux d'abord comme "anti-Pologne», et donc comme antidote à la culture de son pays d'origine et au droit de préemption qu'elle exercerait sur tout esprit qui se veut polonais.

Je voudrais examiner les conséquences d'un tel parti pris sur l'image de la culture tchèque que donne la trilogie de Szczygieł, puis la façon dont les Tchèques, destinataires "occasionnels» et secondaires de cette œuvre, l'ont reçue : ce double point de vue, interne et externe, permettra d'évaluer une entreprise qui fixe les Tchèques dans une image finalement tout aussi instrumentalisée que celle donnée en son temps par le petit reporter belge au pays des Soviets'.

La trilogie tchèque de Mariusz Szczygieł se compose des trois recueils regroupant les reportages qu'il a écrits sur des sujets tchèques: Gottland, Chacun son paradis et Amour céleste. Ce n'est qu'une partie (conséquente) de sa production, partie publiée sous cette forme entre 2006 et 2012 en polonais (entre 2007 et 2012 en tchèque), obéissant à un principe d'organisation interne quasiment maniaque, dont on se rend difficilement compte quand on s'en tient à la version française. D'une part, le dernier volet de la trilogie, Láska nebeská [Amour céleste], n’a pas été traduit en français, et d'autre part, le second volet, Chacun son paradis, sans explication ni de l'éditeur ni de la traductrice, a été amputé de deux reportages et donne ceux qui restent dans un ordre entièrement différent des éditions polonaise et tchèque ${ }^{6}$ - sans parler de la disparition presque

3. M. Szczygieł, Láska nebeská [Amour céleste], trad. H. Stachová, Prague, Jaroslava JiskrováMáj-Dokořán, 20I2, p. I6I : "Polák ve mně vstal z mrtvých». Le sous-titre de cette IVe partie d'Amour céleste, ouvrage paru seulement en polonais et en tchèque, indique : "Opravdu jsem byl dlouho presvědčen, že Polák ve mně trpi impotencí.» [En réalité, je suis convaincu depuis longtemps que le Polonais en moi souffre d'impuissance.]

4. M. Szczygieł, [Je n'écris pas en particulier pour les Tchèques], entretien du I6 décembre $20 \mathrm{I} 2$ avec J. Lojín, art. cité, p. I : "Mám své 'demoralizujici posláni” vưči Polákưm [...].» [J’ai ma propre "mission de démoralisation" vis-à-vis des Polonais.]

5. Hergé, Les Aventures de Tintin, reporter du "Petit Vingtième», au pays des Soviets, TournaiParis, Casterman, I930.

6. Les deux reportages absents de Chacun son paradis (ouvr. cité) sont le neuvième et le quatorzième des vingt et un reportages figurant dans les éditions polonaise et tchèque : «Est-ce 
complète des photos présentes dans les deux livres, sans doute pour des raisons économiques ou de parti pris éditorial.

Arrêtons-nous un instant sur le choix de ces photos et sur leur fonction dans la trilogie. Mariusz Szczygieł fait appel principalement à deux représentants éminents de la photographie tchèque : Pavel Štecha (1944-2004), grand photographe de la vie quotidienne dans la Tchécoslovaquie des années i9681989, fournit six photos pour la version polonaise de Gottland et huit pour la version tchèque; František Dostál, né en 1938, qui nourrit le même goût que Pavel Štecha pour les gens ordinaires et plus encore pour les situations cocasses de la vie de tous les jours ${ }^{7}$, fournit dix photos en noir et blanc pour la version tchèque de Chacun son paradis [Udèlej si ráj], absentes de la version polonaise, et surtout vingt-deux photos, toujours en noir et blanc et le plus souvent inédites, pour Láska nebeská [Amour céleste]. František Dostál est étroitement associé à ce dernier volet de la trilogie comme en témoigne la première partie intitulée "Docela všední obyčejný den» [Un jour tout à fait banal et ordinaire] : c'est en réalité un texte du photographe qui a accepté de compléter cinq amorces de phrases malicieusement soumises à sa sagacité par Mariusz Szczygieł en guise d'hommage ${ }^{8}$.

Toutes ces photos - celles de Pavel Štecha et de František Dostál comme celles des photographes polonais retenus à l'occasion dans les éditions polonaises - ont pour fonction, de l'aveu de l'auteur lui-même, d'illustrer l'humeur dans laquelle les textes sont censés plonger le lecteur' ${ }^{9}$. Et de fait, elles amplifient l'impression produite par les textes auxquels elles donnent un côté plus franchement décalé et humoristique à la fois, tout à fait bienvenu. Ce phénomène

que je pourrais ne pas croire?» [Copak bych mohl nevěrit?] et «Lapám po dechu» [Lapám po dechu], dans Udělej si ráj [Chacun son paradis], Dokořán/Jaroslava Jiskrová-Máj, 20II, p. 82 et p. I43-I49 (version tchèque). Ils se rapportent pour l'un à une enquête sur la foi des jeunes Polonais et pour l'autre à un récit à la première personne que l'on peut raisonnablement attribuer à Milan, policier converti au catholicisme qui apparaît dans «Bon week-end avec le pape!», p. I53-I54 de "Chacun son paradis» (version française). Outre ces deux reportages, il manque aussi dans la version française quelques passages du texte original, sans qu'on en comprenne la raison. La logique qui a présidé à la réorganisation du livre, n’apparaît pas très clairement non plus. Peut-être a-t-on voulu placer en tête les textes censés intéresser davantage le lecteur francophone.

7. F. Dostál est particulièrement connu pour deux cycles de photographies, «Psi a lidé» [Les chiens et les gens] (1972) et "Letní lidé» [Les gens l'été] (I968-1990).

8. M. Szczygieł, Láska nebeská [Amour céleste], ouvr. cité, p. II-I6 (version tchèque).

9. Ibid., p. I4. Dans ce passage, M. Szczygieł qualifie les photographies de Dostál de «nejlepš̌ ilustraci nálady, do niž nás uvedou dvě slova titulu toho knihy" [meilleure illustration de l'humeur dans laquelle nous plongent les deux mots du titre de ce livre], en l'occurrence Láska nebeská [Amour céleste] : je prends la liberté d'appliquer cette remarque à l'ensemble des photos de la trilogie. 
est à vrai dire surtout sensible dans les éditions tchèques, plus encore que dans les éditions polonaises où les photos sont moins nombreuses et n'apparaissent pas dans le même ordre ni au même emplacement, même quand elles sont identiques. Ces différences manifestent d'ailleurs le soin avec lequel ces éditions ont été préparées pour leur public respectif, sur le plan graphique ${ }^{\mathrm{ro}}$, sur le plan du paratexte, mais aussi sur le plan du message, on va le voir.

On observe le même soin scrupuleux dans le montage des textes à l'intérieur de chacun des trois ouvrages, montage qui obéit toujours à une logique précise. Considérons le premier volume de la trilogie, Gottland ${ }^{\text {II }}$. Le titre est pour beaucoup dans le succès du livre : il est une allusion transparente à Karel Gott, le célébrissime crooner tchèque ${ }^{\text {i2 }}$, et aussi au musée ouvert sous ce nom de 2006 à 2008 dans le village de Jevany, à l'est de Prague, dans la villa que le chanteur y a achetée en 1969 (le musée a fermé en raison de difficultés financières, mais il existe un site de visite virtuelle de la villa qui "vous permet de regarder dans la vie de Karel Gott comme si vous la partagiez $\left.{ }^{13} »\right)$. Mais Gottland, c'est aussi littéralement en allemand le "pays de Dieu ", par antiphrase toutefois, puisque la Tchéquie aime à se donner pour la championne toutes catégories de l'athéisme. Or Dieu (Gott) a de fait été remplacé dans ce pays par Karel Gott qui figure, selon Mariusz Szczygieł, «le sacré dans une réalité désacralisée ${ }^{\mathrm{T}} »:$ le culte que les Tchèques vouent au chanteur déclinant, plutôt qu'au Dieu chrétien, viendrait de ce qu' "ensemble [Karel Gott et les Tchèques] ont survécu au communisme ${ }^{15}$ " en composant avec la violence de l'Histoire. C'est donc qu'il y a une autre façon de survivre que celle, héroïque et sacrificielle, des Polonais : en s'adaptant. Gottland présente ainsi dans l'ordre chronologique, de I882 à 2003, le portrait en seize "chapitres» de dix personnalités tchèques, cinq hommes et cinq femmes, dans leur tentative

IO. J. Jiskrová est l'auteur de la conception graphique et de la maquette de la trilogie tchèque de M. Szczygieł, très certainement avec la participation étroite de l'auteur. Un exemple caractéristique de l'adaptation des ouvrages à leur lectorat est la photo due à M. Mutor des "Pisseurs", une œuvre du sculpteur tchèque D. Černý représentant deux hommes en train d'uriner dans un bassin à l'image de la République tchèque : la photo figure à la page 233 de l'édition originale polonaise de Chacun son paradis (Zrób sobie raj, Wolowiec, Éditions Czarne, 20Io) pour illustrer la capacité des Tchèques à se moquer de leur propre pays, mais a disparu de l'édition tchèque au profit des seules photographies de F. Dostál, peut-être aussi à cause de son caractère excessivement provocateur pour un public tchèque.

II. M. Szczygieł, Gottland, Wolowiec, Éditions Czarne, 2006; en tchèque : Gottland, trad. du polonais H. Stachová, Dokořán, J. Jiskrová-Máj, 2007; en français : Gottland, trad. du polonais M. Carlier, Arles, Actes Sud, 2008.

I2. Ibid., p. 185 (version française) : "C'est à la fois le Presley et le Pavarotti tchèque."

I3. <http://muzeum.gottland.eu/> (je traduis).

I4. M. Szczygieł, Gottland, ouvr. cité, p. 193 (version française).

I5. Ibid., p. I94. 
de s'adapter à leur temps, du cordonnier Tomáš Bata, fondateur de l'empire des chaussures Bata, à l'étudiant Zdeněk Adamec qui s'est immolé par le feu sur la place Venceslas en 2003, à l'imitation de Jan Palach en $1969^{16}$. Les personnalités évoquées ne sont donc pas seulement des intellectuels, mais elles ont toutes été dans l'histoire tchèque des objets médiatiques, ce qui entraîne tout de même une surreprésentation relative du monde de la culture (cinéma, chanson, littérature, arts plastiques).

Dans un entretien de 2006 avec le journaliste allemand Volker Hummel, "Un bon reportage fonctionne comme un roman ", Mariusz Szczygieł explique la méthode qui est la sienne par exemple dans Gottland: il écrit d'abord la première et la dernière phrase de chaque reportage, puis les relie à travers une dramaturgie ${ }^{17}$. Cette dramaturgie implique l'évocation de la famille, des amis, des destins proches, semblables ou opposés, de chaque personnalité, si bien que le lecteur croise la route d'une foule de gens, plus ou moins connus, foule qui donne de la chair et de la vie aux portraits principaux. D'autant que tous les chapitres sont reliés entre eux par des liens thématiques discrets, qu'il s'agisse d'un lieu (le Palais Lucerna, place Venceslas à Prague, ou le monument de Staline dans le parc de Letná dont l'emplacement est visible depuis les fenêtres de la nièce de Kafka), d'un scénario de film, d'un livre (La Métamorphose), d'une situation commune (quelqu'un est confronté à la divulgation d'informations concernant sa vie privée sur les ondes ou dans la presse), d'une figure historique (Jan Hus ou Jan Masaryk), etc. L'auteur joue de ces liens pour créer des effets de rythme : il retarde l'information en la fractionnant sur plusieurs chapitres ou procède au contraire à des raccourcis au gré d'associations de noms et d'idées astucieuses. Un autre trait de disposition contribue à la fois à l'unité et à la variété de l'ensemble : l'alternance savante des chapitres longs et des chapitres courts qui donne au livre sa respiration propre, en quelque sorte.

Et tout comme entre chapitre long et chapitre court, on balance, à la lecture de ces portraits, entre des sentiments opposés : l'admiration et l'exaspération, la compassion et l'antipathie. Si la thèse est claire (l'héroïsme est un luxe que

I6. Dans l'ordre chronologique adopté par Gottland: Tomáš Bata, Lída Baarová, Otakar Švec, Věra Saudková, Jan Procházka, Helena Vondráčková, Marta Kubišová, Edvard Kirchberger / Karel Fabián, Zdeněk Adamec, Jaroslava Moserová. Le destin de cette dernière, décédée en 2006, fournit le contrepoint de celui d'Adamec.

17. M. Szczygieł, "Eine gute Reportage funktioniert wie ein Roman», entretien du 20 avril 2006 avec V. Hummel pour le journal en ligne Netzzeitung <https://web.archive.org/ web/20060425044345/>, <http://www.netzeitung.de/voiceofgermany/393320.html> [consulté le 24/08/15] : "Am Anfang stehen bei mir immer der erste und der letzte Satz des Textes, die muss ich zuerst finden. Dann suche ich nach einer Dramaturgie, die mich vom ersten zum letzten Satz führt, die sie miteinander verbindet.» 
ne peuvent s'offrir les Tchèques en raison de leur faible nombre $\left.{ }^{18}\right)$, le livre se préserve du manichéisme en présentant des personnalités certes toutes victimes de leur temps à des titres divers, mais dont les réactions mêlent, à des degrés divers aussi, courage et lâcheté, orgueil et désespoir, bêtise et générosité. Ce sont des gens "normaux» qui nous sont présentés là, avec leurs misères et leur grandeur : Szczygieł choisit de regarder les drames de l'histoire à l'échelle de ces destins singuliers, estimant «que les gens ont grand besoin de livres sur l'histoire, mais pas faits par un historien; de livres comme les miens où la grande Histoire sert seulement de toile de fond à la vie de l'homme concret ${ }^{19}$ " - l'homme concret qui fait le gros dos en attendant que les choses se passent, comme les deux personnages poliment ordinaires du dessin de Bape (Jiř́ Bartoš et Vladimír Pergler), publié dans le journal satirique Dikobraz en décembre I968 après l'écrasement du printemps de Prague et reproduit dans Gottland ${ }^{20}$ : "Joyeux Noël... en I989". Un souhait aussi banal prend évidemment des allures de prophétie à la lumière des événements de novembre 1989; or la veuve du dessinateur Vladimír Pergler jure que c'est une simple erreur de date, attitude que Szczygieł attribue à la modestie congénitale des Tchèques qui n’imaginent pas avoir prise sur l'histoire, fût-ce par le biais d'un simple don de double vue ${ }^{2 r}$.

Chacun son paradis [Udèlej si ráj], le second volet de la trilogie, s'inscrit dans la continuité de Gottland en développant un trait caractéristique du monde tchèque selon l'auteur, que Gottland n'avait fait que signaler : l'absence de Dieu

I8. Cette thèse est mise symboliquement dans la bouche prestigieuse de la journaliste dont le nom est attaché à celui de Kafka, M. Jesenská (et non Jasenská, comme l'écrit malencontreusement la traductrice française à plusieurs reprises), dans Gottland, ouvr. cité, p. 72-73 (version française) : "Il est sans doute glorieux de verser son sang pour sa patrie dans un sursaut d'héroïsme. Je pense même que cela n'est pas bien difficile. Mais nous, nous devons agir différemment. Nous devons vivre. Nous devons épargner chaque être humain parmi les nôtres, chaque force vive, si petite soit-elle. Nous ne sommes pas assez nombreux pour nous permettre de faire des gestes héroïques. Nous sommes huit millions - c'est peu, trop peu pour commettre des suicides. Mais suffisamment pour vivre."

19. M. Szczygieł, «Nepíšu speciálně pro Čechy» [Je n’écris pas en particulier pour les Tchèques], entretien du I6 décembre 2012 avec J. Lojín, art. cité, p. 3 : "Myslím, že lidé moc potrebuji knihy o historii, ale ne od historika, ale takové, jako jsou mé, kde se velká historie jeví pouze jako pozadí života konkrétního člověka.» (Trad. M.-O. Thirouin) Mariusz Surosz est-il visé par cette déclaration? L'historien polonais, auteur de Pepici (Pepik est le diminutif du prénom Joseph par lequel les Polonais désignent leurs voisins tchèques avec une certaine condescendance), ouvrage traduit sous ce titre par P. Weigel aux Éditions Plus en 20II, y examine en effet d'un point de vue critique un certain nombre de destins tchèques (Jan Masaryk, Emil Hácha, Klement Gottwald, Jaroslav Seifert, Pavel Tigrid, Alois Eliáš, K. H. Frank, Milada Horáková, František Kriegel, Milena Jesenská, Jan Patočka, Toyen) en mettant davantage l'accent sur le contexte politique et historique que ne le fait Szczygieł dans Gottland.

20. M. Szczygieł, Gottland, ouvr. cité, p. 198 (version française). La traductrice a malencontreusement traduit le titre du reportage de Szczygieł commentant ce dessin par «Bonnes fêtes».

2I. Ibid., p. I99-20I. 
et de la religion chrétienne dans la vie des «Tchèques de maintenant ${ }^{22}$ ». Ce trait est dans l'esprit de Szczygieł lié à la capacité des Tchèques à vivre « légèrement ", c'est-à-dire à ne rien prendre au sérieux et à rire de tout, comme y invite l'injonction qui sert de titre au livre (malheureusement effacée de la traduction française) : fais-toi ton paradis! "Tandis que pour les Polonais», résume un critique tchèque, «le Paradis est donné par Dieu une fois pour toutes, le Tchèque doit apprendre et apprend de fait à se créer son propre paradis ${ }^{23} »$. La question se pose de savoir comment, dans ces conditions, les Tchèques affrontent le scandale des scandales, la mort. Précisément en élevant la vie quotidienne au rang de la poésie, en en faisant une fête de tous les instants, attitude volontariste dont la culture tchèque (Bohumil Hrabal en tête) serait le reflet exact, selon Szczygieł : pour lui, la culture tchèque fonctionne à la manière d'un véritable antidépresseur; c'est "du Prozac ${ }^{24}$ », apte à compenser les effets délétères d'une vie sans Dieu. Le rire des Tchèques n'est donc pas forcément un rire plébéien et vulgaire, une petitesse d'âme comme le croient trop souvent les Polonais; c'est plutôt le masque modeste que revêtirait par discrétion "le désarroi tragique ${ }^{25}$ " de consciences privées de la foi consolatrice en une vie après la mort.

L'hédonisme tchèque constituerait ainsi une agréable alternative au goût du malheur et à la nécrophilie des Polonais ${ }^{26}$, mais plus largement aussi à la "morosité de notre époque», selon la formule que Szczygieł dit emprunter à Michel Houellebecq ${ }^{27}$ : telle est donc la perspective générale dans laquelle

22. M. Szczygieł, Zrób sobie raj, Wolowiec, Éditions Czarne, 2oıo; Udělej si ráj, trad. du polonais H. Stachová, Dokořán, J. Jiskrová-Máj, 20ıI; Chacun son paradis, trad. M. Carlier, Arles, Actes Sud, 20I2. Ici : p. 7 (version française).

23. J. M. Heller, "Mariusz Szczygieł : Udělej si ráj», recension du 28 juin 201 publiée dans la revue électronique iLiteratura: "Zatímco Polákìm byl ráj dán od Boha jednou provždy, Čech si musi umèt a umi svioj vlastni ráj vytvorit." (Je traduis.) Voir <http://www.iliteratura.cz/ Clanek/28533/szczygie-mariusz-udelej-si-raj> [consulté le 24/08/2015].

24. M. Szczygieł, Chacun son paradis, ouvr. cité, p. 97 (version française).

25. M. Szczygieł, Udělej si ráj, ouvr. cité, p. 7 (version tchèque) : "Udělej si ráj je mimo jiné vyprávènim o tom, co mě fascinovalo v české kultuře. O smíchu jako masce. Někdy masce tragické bezradnosti.» (Je souligne.) Cette citation est extraite d'un texte, "Místo úvodu» [En lieu et place d'introduction], qui ne figure que dans l'édition tchèque puisque Szczygieł s'y adresse directement à son "cher lecteur tchèque».

26. Voici un exemple de considérations de Szczygieł illustrant ce point de vue et figurant dans la version française de Chacun son paradis, ouvr. cité, p. 208-209 : "j'ai cité le poète polonais Norwid - "la Pologne, ce n'est que de la mémoire et des tombes" - ce qui a provoqué un éclat de rire chez le public praguois [...]. Pour vivre, notre nation a besoin de malheur. [...] La culture polonaise est une culture nécrophile. [...] Les Polonais savent pourtant très bien s'unir, mais seulement dans le malheur".

27. M. Szczygieł, Udělej si ráj, ouvr. cité, p. 7 (version tchèque) : «Pokud naši dobu - jak trvdi Michel Houellebecq - nejlépe charakterizuje slovo 'pochmurnost', pak právě Češi dovedou tuto pochmurnost impozantnim zpuisobem odstranit ze zorného pole." 
s'inscrivent les vingt et un «chapitres» de Chacun son paradis. Ce livre est néanmoins d'une facture assez différente de Gottland: il s'apparente moins à une série de reportages qu'à un montage de textes de nature différente, privilégiant tantôt le document brut, tantôt l'anecdote (personnelle ou rapportée), le récit d'interview ou d'enquête, les réflexions d'ordre général ${ }^{28}$... Le cœur en est les trois chapitres (IO, I2 et I4) consacrés à l'enquête sur les liens des Tchèques avec la religion catholique ${ }^{29}$, enquête menée sur place et par mail. Cette partie centrale est précédée de sept chapitres (2 à 8) et suivie de sept autres (I5 à 2I), respectivement sur les causes et les effets de l'athéisme tchèque dans la vie de tous les jours et dans la culture propre au pays ${ }^{3 \circ}$. Cette construction peut se comprendre comme un hommage discret au Livre du Rire et de l'Oubli de Milan Kundera, dont l'ombre accompagne Chacun son paradis, tant sur le plan thématique (le rire) que formel (le principe de la variation sur un ton de léger persiflage ${ }^{31}$. Kundera, dont le nom n'apparaît que furtivement au détour d'une citation malignement dépourvue de source ${ }^{32}$, ne fait pas explicitement partie du panthéon personnel de Mariusz Szczygieł, mais ce dernier a sans doute été frappé par la similitude de leur trajectoire, toutes proportions gardées, qui les arrache à leur nation d'origine. À ceci près que cette trajectoire emmène Szczygieł dans le pays qu'a fui Kundera, ce "pays-paradis ", aux yeux

28. J. M. Heller, «Mariusz Szczygieł : Udělej si ráj», art. cité : «Je to esej kombinovaný s postupy dokumentárni literatury, žánrová smès úvah, reportáži, črt z náhodných setkáni, reflexi, rozhovorü." 29. C'est ce que fait remarquer P. Zídek dans sa recension du I ${ }^{\mathrm{er}}$ mai 20II, "Szczygiet udělá pointu i ze smrti» [Szczygieł ménage un effet de surprise avec la mort-même], sur Lidové noviny: $<$ http://www.lidovky.cz/szczygie-udela-pointu-i-ze-smrti-do9-/kultura.aspx?c=AıIO429_I5I349_ ln_kultura_wok> [consulté le 24/08/20I5].

30. Je dote ici de numéros les reportages dans l'ordre donné par les versions polonaise et tchèque. Les reportages non cités (I, 9, II et I3) fonctionnent comme des articulations ou des variations à l'intérieur de la structure globale.

31. Voir M. Kundera, Le Livre du Rire et de l'Oubli, Paris, Gallimard, coll. «Folio », 1979, p. 268 : "Tout ce livre est un roman en forme de variations. Les différentes parties se suivent comme les différentes étapes d'un voyage qui conduit à l'intérieur d'un thème, à l'intérieur d'une pensée, à l'intérieur d'une seule et unique situation dont la compréhension se perd pour moi dans l'immensité.» Cette description convient parfaitement à Chacun son paradis.

32. M. Szczygieł, Chacun son paradis, ouvr. cité, p. 48 (version française) : " "La lutte de l'homme contre le pouvoir est une lutte de la mémoire contre l'oubli", déclarait, en I97I, un des personnages de Milan Kundera. " La citation est précisément extraite du Livre du Rire et de l'Oubli, ouvr. cité, p. I4 : «On est en I97I et Mirek dit : la lutte de l'homme contre le pouvoir est la lutte de la mémoire contre l'oubli. »Curieusement, ce roman de Kundera ne figure pas dans la liste des «livres cités, évoqués et décrits» présente à la fin de la version polonaise et de la version tchèque de Chacun son paradis, où l'on ne trouve mention que d'Une rencontre, le dernier essai en date de Kundera paru aux Éditions Gallimard en 2009 (Udělej si ráj [Chacun son paradis], ouvr. cité, p. 219 [version tchèque]). 
du Polonais, dont l'hymne national invite à rester couché plutôt qu'à prendre les armes pour le défendre ${ }^{33}$.

Car en Tchéquie, on ne vous demande même pas de croire en votre pays : la preuve vivante en est le sculpteur à succès, sinon talentueux, David Černý, auteur du Christ en pièces détachées qui illustre la couverture de l'édition polonaise de Chacun son paradis, un «intellectuel d'une rare vulgarité» selon les termes d'un critique polonais rapportés par Mariusz Szczygieł34. Or Černý est à ses yeux l'incarnation la plus radicale de la liberté faite aux Tchèques de dire du mal de leur pays, encore une fois à la différence des Polonais : pour Černý, l'identité nationale tchèque, c'est par exemple "une masse blanche inintéressante, fade, gavée de knedlíky et imbibée de bière» et le Tchèque de souche "un beauf rapiat, roublard, retors et un peu lâche ${ }^{35}$ ». Ce sens patriotique à rebours, qui suscite la surprise et l'envie de Mariusz Szczygieł, a par exemple inspiré le groupe des «Pisseurs», créé par Černý à l'occasion de l'adhésion de la République tchèque à l'Union européenne et montrant deux hommes urinant dans un bassin qui a la forme du pays ${ }^{36}$. Signe des temps, pour Szczygieł, qui considère que les patriotismes sont voués à disparaitre et espère qu'on pourra bientôt choisir son pays à l'instar de n'importe quel produit commercial ${ }^{37}$. Pourtant, Szczygieł est conscient des limites de l'attitude qui consiste à refuser systématiquement le pathos et à rire de tout. Car qu'est-ce, sinon une manière paradoxale de fuir le réel au lieu de s'y engager, donc aussi une forme de lâcheté et de cynisme commode, comme Szczygieł charge l'écrivain tchèque Jan Faktor de le rappeler ${ }^{38}$ ? Sans compter que sur le plan esthétique, et non plus moral cette fois-ci, le goût navrant des petits plaisirs de la vie quotidienne n'est jamais très loin du mauvais goût tout court.

C'est pourquoi le troisième volet de la trilogie cherche à montrer que, malgré tout, le Tchèque sait s'élever au-dessus du réel sans sombrer dans un relativisme nihiliste : il s'agit de Láska nebeská [Amour céleste], dont le titre original est en tchèque (avec les signes diacritiques, précise Szczygieł ${ }^{39}$ ) pour «réjouir» le lecteur polonais tant par la couleur bleue que par l'allusion obscène

33. M. Szczygieł, Chacun son paradis, ouvr. cité, p. II-I3 (version française).

34. Ibid., p. 20 (version française).

35. Ibid., p. 28 (version française).

36. Voir n. Io.

37. M. Szczygieł, Chacun son paradis, ouvr. cité, p. I9 (version française).

38. Ibid., p. 229.

39. M. Szczygieł, Láska nebeská [Amour céleste], ouvr. cité, p. 6 (version tchèque). Szczygieł commente le choix de son titre dans un texte destiné encore une fois au seul lecteur tchèque sous le titre de "Dopis českému čtenáři» [Lettre au lecteur tchèque], ibid., p. 5-8. La version polonaise est, quant à elle, parue en effet sous le titre de Láska nebeská quelques mois plus tôt, en janvier 20I2, aux éditions du groupe de presse Agora à Varsovie. 
qu'il connote dans sa langue maternelle ${ }^{40}$. L'ouvrage, consacré à la littérature tchèque, rend hommage à son "amour du détail, de la vie et de l'homme ordinaire» qui "sublime la banalité» des choses, sachant qu'un tel sens de «la grâce du réel» ne peut lui venir que de cette «absence de Dieu et d'une quelconque perspective d'éternité» si typiquement tchèque ${ }^{4 \mathrm{r}}$ (on voit le lien avec le volet précédent de la trilogie) : car l'inexistence de Dieu rend infiniment précieuse la seule réalité que l'on possède, aussi banale soit-elle. L'esprit de la littérature tchèque tiendrait donc dans ce dicton insupportable aux Polonais, mais qui ravit Szczygieł : « tant que tu vis, mange et bois; après la mort, tu n’auras plus d'occasion de réjouissance ${ }^{42}$ » «La littérature tchèque, c'est mon amour céleste à moi ", résume-t-il dans une nouvelle profession de foi paradoxale ${ }^{43}$.

Láska nebeská [Amour céleste] reproduit, à la suite de cette introduction ${ }^{44}$, les dix-sept articles commandés à Mariusz Szczygieł à l'occasion de la publication en polonais, aux Éditions Agora, de seize œuvres littéraires tchèques du $\mathrm{Xx}^{\mathrm{e}}$ siècle, écrites par treize auteurs différents, accompagnées chacune d'un DVD avec le film qui en a été tiréts. Chaque article se présente comme une

40. Szczygieł explique dans la version tchèque de Láska nebeská [Amour céleste], ouvr. cit, p. 6-7, que laska signifie en polonais bâton, mais désigne aussi en langage familier une jolie fille ou l'organe sexuel masculin. Pour les Tchèques, le titre du livre évoque la chanson sirupeuse interprétée par Waldemar Matuška et Eva Pilarová, «tube» de la Tchécoslovaquie des années I960 resté dans toutes les mémoires: Ach, ta láska nebeská.

4I. Ibid., p. 20 (version tchèque) : «Česká literatura miluje detail, život a obyčejného člověka. Často povyšuje všednost. [...] Ono čerpáni puivabu z v̌sednosti života, viditelně jak v české literatuře, tak ve filmu, musi podle mého názoru pramenit z nedostatku Boha a perspektivy věčnosti. Máme jen jeden život, žádný posmrtný nebude, a tak ho musime maximálně využit a zhodnotit." (Trad. M.-O. Thirouin.)

42. Ibid., p. I9 (version tchèque) : «Dokud žiješ, jez a pij, po smrti užžádnou radost mít nebudeš». Ce dicton, affiché dans un café à la frontière tchéco-polonaise, choque tant les clients polonais que le patron a été obligé de l'enlever, selon le récit qu'en fait Szczygieł (ibid., p. 19-24).

43. Ibid., p. 7 (version tchèque) : «'Česká kuchyně má Čecha držet prí zemi. Česká literatura ho má povznést k nebi. Česká literatura - to je moje láska nebeská." [La cuisine tchèque est censée maintenir le Tchèque sur terre. La littérature tchèque est censée l'élever au ciel. La littérature tchèque, c'est mon amour céleste à moi.]

44. Rappelons que le livre s'ouvre sur le texte du photographe F. Dostál dont il a été question plus haut : l'introduction de Szczygieł sur la littérature tchèque constitue ainsi le début de la deuxième partie de l'ouvrage, recueil des dix-sept articles dont il est question maintenant.

45. Szczygieł présente successivement: B. Hrabal, Obsluhoval jsem anglického krále [Moi qui ai servi le roi d'Angleterre]; O. Pavel, Smrt krásných srncu [La Mort des beaux chevreuils]; J. Papoušek, Černý Petr [L’As de pique] ; Z. Svěrák, Vratné lahve [Bouteilles consignées] ; B. Hrabal, Ostře sledované vlaky [Trains étroitement surveillés]; V. Vančura, Rozmarné léto [Un été capricieux]; J. Hašek, Osudy dobrého vojáka Švejka za světové války [Les Aventures du brave soldat Chvéik pendant la guerre mondiale : parties I et II, puis parties III et IV]; V. Havel, Žebrácká opera [L'Opéra de quat'sous]; B. Hrabal, Slavnosti sněženek [Les Fêtes des perce-neige]; B. Hrabal, Perlička na dně [Petites Perles au fond de l'eau] ; J. Brdečka, Limonádový Joe [Jo limonade]; L. Grosman, Obchod na korze [Le Magasin de la Grand-Rue = Le Miroir aux alouettes]; J. Škvorecký, Tankový prapor 
«improvisation» (jam session) autour d'une phrase retenue par Szczygieł dans chacun des livres, le critère étant que cette phrase doit fournir aux Polonais un angle de vue inattendu sur l'œuvre concernée, tout en illustrant la thèse selon laquelle la littérature tchèque aime «le détail, la vie et l'homme ordinaire». Ce qui intéresse les Polonais, selon Szczygieł, c'est la façon qu'ont les Tchèques, à leur différence, de s'amuser de tout, y compris du conformisme, de la bêtise et de leurs propres faiblesses ${ }^{46}$.

Le livre ne s'arrête pourtant pas là : il finit par une pirouette qui se présente aussi comme la conclusion générale de l'entreprise commencée avec Gottland. Szczygieł rapporte en effet, dans la troisième partie de Láska nebeská [Amour céleste], intitulée "Ma vie à Gottland ${ }^{47}$ ", une série de quatre anecdotes déceptives qui relativisent les thèses exposées depuis Gottland à propos de la nation tchèque, constituée en anti-Pologne : il y est question d'échec, de malentendu, de critiques reçues de la part des Tchèques eux-mêmes. Le processus se poursuit dans la quatrième et dernière partie de Láska nebeská [Amour céleste], «Le Polonais en moi s'est relevé d'entre les morts", dont il a déjà été question ${ }^{48}$, en ramenant l'auteur à ses origines, plus profondément inscrites en lui qu'il ne le pensait, c'est-à-dire à la Pologne.

Cette fin auto-ironique, qui renvoie l'auteur là d'où il vient, n'est pas dépourvue d'élégance, mais elle ne laisse pas d'intriguer : est-ce une simple pose, un accès de (fausse) modestie, l'expression d'un remords, réel ou feint? La constitution de la Tchéquie en anti-Pologne au fil de ces trois livres était pourtant une entreprise "d'idéalisation " pleinement assumée. Szczygieł admet à plusieurs reprises avoir procédé à une stylisation de la réalité tchèque dont il a sélectionné les traits qui lui convenaient, conformément à sa mission qui est de susciter la réflexion (des Polonais) à l'échelle du microcosme, du détail apparemment insignifiant à partir duquel il généralise. D'autant qu’il prend grand soin, on l'a vu, à la fois d'éviter le manichéisme et de souligner la cohérence globale de son entreprise, à l'échelle du recueil comme de la trilogie tout entière. Et si l'on ajoute à cela le travail de documentation considérable auquel s'est livré l'auteur, incollable ou presque sur la culture tchèque qu'il connaît de l'intérieur ${ }^{49}$, on ne peut qu'admirer le résultat obtenu. On regrette à cet égard

[L'Escadron blindé]; L. Fuks, Spalovač mrtvol [L'Incinérateur de cadavres]; K. Čapek, Krakatit; M. Viewegh, Výchova divek v Čechách [L'Éducation des jeunes filles en Bohême].

46. M. Szczygieł, Láska nebeská [Amour céleste], ouvr. cité, p. 23 (version tchèque).

47. Ibid., p. I29-160: "Mĩj životv Gottlandu».

48. Ibid., p. I6I-I70. Voir n. 3.

49. J'ai repéré en ce qui me concerne une seule véritable erreur dans un ensemble d'une grande érudition : dans Chacun son paradis, ouvr. cité, p. 224 (version française), Szczygieł attribue à R. Denemarková l'honneur d'avoir écrit le premier roman tchèque sur l'expulsion des 
que l'éditeur français ait jugé bon de faire disparaître les bibliographies qui figurent à la fin des volets I et 2 de la trilogie, jugeant sans doute qu'un lecteur francophone n'aurait pas l'usage de références en polonais et en tchèque, c'est une erreur, car celles-ci mettent en évidence le travail effectué par Szczygieł entre la collecte des informations et leur intégration dans des textes ramassés, elliptiques, qui jettent des ponts entre le particulier et le général.

On identifie d'ailleurs très bien, au milieu d'une telle érudition, les figures tutélaires qui guident l'auteur dans sa tâche, la «trinité pragoise » à laquelle est voué "pan Mariusz». Il faut citer en premier lieu le roi des reporters, le très marxiste Egon Erwin Kisch (1885-1948), auteur de trois recueils de reportages sur sa ville natale, Les Rues et les Nuits de Prague, Enfants de Prague et Aventures pragoises $^{50}$. Kisch est mort alors qu'il venait de commencer un reportage sur la dynastie Bata, raison pour laquelle Mariusz Szczygieł lui dédie son propre reportage sur le sujet ${ }^{5 \mathrm{I}}$. Ce dernier entretient en outre - détail purement anecdotique - une relation de grande complicité et amitié avec sa traductrice tchèque, Helena Stachová, qui n'est pas sans rappeler celle qu'entretenait Kisch avec sa propre traductrice, Jarmila Haasová-Nečasová (1896-1990). Kisch correspond à la figure de Dieu le Père dans la trinité personnelle de l'écrivain polonais dont il est le modèle, sur le plan esthétique (l'idée de fantaisie logique pour voir derrière les choses), sur le plan moral (l'engagement, l'intérêt pour les marginaux) et sur le plan politique (l'homme de gauche). Dans le rôle du Fils, on imagine assez bien Egon Bondy (1930-2007), poète et philosophe marxiste anti-stalinien qui a choisi un nom juif en 1949 pour protester contre les purges antisémites alors en cours à Prague. C'est le roi de l'underground tchèque musical et littéraire, un dissident et un marginal radical, qui a payé de sa personne jusqu'à mourir en mettant le feu à son lit avec une cigarette mal éteinte, à Bratislava, ville où il s'était installé en 1993 pour protester contre la partition de la Tchécoslovaquie ${ }^{{ }^{2}}$. Dans cette trinité enfin, le rôle de l'Esprit est indiscutablement tenu par Jára Cimrman, un personnage de fiction inventé en 1966 par Jiří Šebánek et Zdeněk Svěrák, si populaire qu’il a failli emporter en 2005 le titre de "plus grand Tchèque de tous les temps" soumis au vote

Allemands de Tchécoslovaquie après 1945 (Penize od Hitlera [L'Argent d'Hitler], Host, 2006), oubliant le roman de J. Durych, Boži duha [L'Arc-en-ciel de Dieu], achevé en 1955 et publié en 1969.

50. E. E. Kisch, Aus Prager Gassen und Nächten (1912), Prager Kinder (1913), Abenteuer in Prag (1920) : l'ensemble de ces textes est disponible en un volume paru au Aufbau-Verlag de Berlin-Est en 1968.

5I. Voir la dédicace dans M. Szczygieł, Gottland, ouvr. cité, p. 9 (version française).

52. M. Szczygieł, Chacun son paradis, ouvr. cité, p. I6I-I85 (version française) : «Un lit prend feu». 
du peuple tchèque : Cimrman incarne le génie tchèque, c'est-à-dire un génie malheureux, qui arrive toujours après la bataille; il fascine Mariusz Szczygieł parce qu'il illustre à la fois le patriotisme paradoxal des Tchèques, leur capacité à l'autodérision et surtout leur goût de la mystification que lui-même aime à cultiver ${ }^{53}$.

Que risquerait notre auteur polonais en restant sous la protection de sa trinité pragoise plutôt que de regagner le camp polonais? Pas grand-chose si l'on en juge par le succès qu'il remporte en Tchéquie. Le site Internet de l'éditeur tchèque, Dokořán, annonce qu'il en est en septembre 2015 au vingtième retirage de Gottland, ouvrage qui occupe encore, huit ans après sa publication, la cinquième place sur la liste de ses meilleures ventes; le site répertorie également 42 recensions en tchèque du livre qui est aussi disponible en version électronique et sur CD. On sait enfin que Gottland a suscité deux adaptations théâtrales en 20II, à Ostrava et à Prague ${ }^{54}$, et en 2014 un film tourné par cinq étudiants de la FAMU (Filmová a televizní fakulta Akademie múzických umění v Prazess) qui ont, chacun, adapté à l'écran un des reportages du livre ${ }^{56}$. Aujourd'hui encore, ce dernier fait régulièrement l'objet de lectures publiques à Prague. Même si le succès des deux autres volets de la trilogie a été de moindre ampleur, Mariusz Szczygieł est omniprésent dans les médias tchèques où il a multiplié les interviews et les apparitions. Lui-même revendique de un à cinq mails par jour en provenance de Tchéquie ${ }^{57}$.

Mais qui lit Mariusz Szczygieł en Tchéquie? N'ayant trouvé personne dans ce cas parmi mes connaissances, je me suis rabattue sur les commentaires des

53. M. Szczygieł, Láska nebeská [Amour céleste], ouvr. cité, p. I47-I53 (version tchèque) : «Nic $k$ smichu» [Pas de quoi rire].

54. Kdo neskáče, neni Čech! [Qui ne saute pas, n’est pas Tchèque!], au Národni divadlo Moravskoslezské à Ostrava (janvier 20II), mise en scène de Jan Mikulášek, et Masky tragické bezradnosti [Masques du désarroi tragique] au Švandovo divadlo de Prague deux mois plus tard, mise en scène de Petr Štindl. Voir un exemple de critique comparée des deux spectacles sur $<$ http://www.topzine.cz/inscenace-gottland-stejna-hra-ale-dve-ruzna-zpracovani-ktere-je-lepsi> [consulté le 26/08/20I5].

55. Faculté du cinéma et de la télévision de l'Académie des arts du spectacle de Prague.

56. Gottland, film expérimental de Ios minutes, tourné par Klára Tasovská, Rozálie Kohoutová, L. Kokeš, V. Č́ananyová, P. Hátle, qui ont adapté respectivement les reportages sur Zdeněk Adamec, la statue de Staline, la dynastie Bata, l'écrivain E. Kirchberg et l'actrice L. Baarová. Le film a été présenté à la gare de Žižkov à Prague en août 20I4, puis au festival du film de Varsovie en novembre 20I4. Il a été précédé par un faux sondage sur la statue à ériger sur la colline de Letná, là où se dressait Staline jusqu'en 1962 : les 120 ooo participants au sondage ont désigné dans l'ordre Jiřina Bohdalová, Jaromír Jágr et... Karel Gott.

57. M. Szczygieł, "Nepíšu speciálně pro Čechy», [Je n’écris pas en particulier pour les Tchèques], entretien du I6 décembre 2012 avec J. Lojín, art. cité, p. 5 : «Každý den mi prícházejí jeden až pèt mailů od Čechủ. Trvá to užpèt let. " [Chaque jour, me parviennent entre un et cinq mails de la part de Tchèques. Cela fait déjà cinq ans que ça dure.] 
internautes, pour comprendre la nature de l'engouement affiché des Tchèques pour l'auteur polonais. Les commentaires élogieux dominent en effet, et parmi eux, l'éloge le plus fréquent concerne l'euphorie éprouvée à se reconnaitre dans le miroir tendu par un Polonais. Quant aux critiques négatives, elles sont exactement symétriques : les commentateurs se plaignent soit de l'ennui éprouvé à la lecture de choses qu'ils connaissent déjà parfaitement, soit du sentiment de dépression qu'ils retirent du tableau dressé de leur pays par l'écrivain polonais.

Ces deux critiques ont profondément touché Mariusz Szczygieł : j'en veux pour preuve le paratexte destiné aux Tchèques dans les deuxième et troisième volets de la trilogie, sous forme de lettres au lecteur tchèque ${ }^{{ }^{8}}$, où il demande pardon de devoir énoncer des évidences indispensables pour le lecteur polonais, mais bien sûr fastidieuses pour tout habitant de la Tchéquie :

Je n'écris rien spécialement pour les Tchèques. Quand j'écris sur la Tchéquie, je ne pense absolument pas au lecteur tchèque. Si j’y pensais, je n'écrirais rien. [...] Mes livres sont pour les Tchèques seulement DE MANière OcCasionnelLe. Ils sont avant tout pour les Polonais et ensuite pour les Européens.

dit encore Szczygieł dans son entretien de 2012 avec Jiří Lojín 59.

Comment expliquer alors le succès phénoménal de Gottland en Tchéquie, succès que l'auteur accueille visiblement avec plaisir? Il y a là avant tout, me semble-t-il, un effet médiatique qui exploite les indéniables qualités formelles du livre pour vendre un produit proposant en réalité une image stéréotypée des Tchèques, dans laquelle une certaine population se plaît à se reconnaître. "Vous ne vous intéressez qu’à vous-mêmes", lance d'ailleurs Mariusz Szczygieł, légèrement irrité de l'insuccès relatif de ses autres livres en Tchéquie ${ }^{60}$.

58. M. Szczygieł, Chacun son paradis [Udělej si ráj], ouvr. cité, «Místo úvodu» [En lieu et place d'introduction], p. 7-Io (version tchèque), et Láska nebeská [Amour céleste], ouvr. cité, "Dopis českému čtenáŕi» [Lettre à mon lecteur tchèque], p. 5-8 (version tchèque).

59. M. Szczygieł, "Nepíšu speciálně pro Čechy» [Je n'écris pas en particulier pour les Tchèques], entretien du I6 décembre $20 I 2$ avec J. Lojín, art. cité, p. 4 : «Speciálně pro Čechy nic nepišu. Když pišu o Česku, vỉbec na českébo čtenáre nemyslim. [...] Mé knihy jsou pro Čechy pouze PRÍLEŽITOSTNĚ. Jsou predevšim pro Poláky a pak pro Evropany.» (Trad. M.-O. Thirouin.) 6o. Ibid., p. 3-4: "Z mých knižek se u vás nejlépe prodává ta o Céšich, protože rádi čtete o sobè. Zaexperimentovali jsme s českým vydáním mé knihy o ženách, ale ne českých, pouze polskýchvyšla kniha Libuistka. A myslim si, že o ni nebyl velký zájem. Zajimáte se, ale sami sebou. Zatimco napríklad v Itálii se Libuistka prodává nejlépe ze všeho, co jsem napsal. " [ "De tous mes livres, c'est celui sur les Tchèques qui se vend le mieux chez vous parce que vous aimez lire des choses sur vous. Nous en avons fait l'expérience avec l'édition tchèque de mon livre sur les femmes, mais qui n'est pas sur les femmes tchèques, seulement sur les polonaises : Libuistka est donc sorti. Je pense que ce livre n'a pas suscité un grand intérêt. Vous ne vous intéressez qu'à vous-mêmes. Tandis que par exemple en Italie, c'est Libuistka qui se vend le mieux de tout ce que j'ai écrit.» (trad. M.-O. Thirouin) Voir aussi la recension de Gottland par Petr Vaněk du 22 décembre 2007 pour la revue électronique iLiteratura, <http://www.iliteratura.cz/Clanek/2I937/szczygie- 
Pour revenir à la dépression que la lecture de ses livres éveille chez certains Tchèques, elle est le pendant involontaire de la démoralisation visée du côté des lecteurs polonais. Mariusz Szczygieł n'en aime guère l'idée, comme on s'en rend compte en visionnant l'interview qu'il a donnée à une chaîne de télévision tchèque du net, DVTV [Drtinová Veselovský TV], le I9 août $2014^{61}$ : face au journaliste Martin Veselovský qui évoque la possibilité d'un effet déprimant de ce qu'il écrit sur le lecteur tchèque, Szczygieł a du mal à contenir son déplaisir et rétorque que c'est le lecteur qui projette sa dépression sur le livre, et non le livre qui suscite la dépression. En l'écoutant, on se demande brusquement si ce n'est pas plutôt la dépression de l'auteur qui se cristallise dans l'image donnée des Tchèques et de la culture tchèque. Car il est un personnage de plus en plus souvent convié dans la trilogie, comme d'autres convient leur confesseur : c'est le psychothérapeute de Mariusz Szczygieł. La quatrième de couverture de Láska nebeská [Amour céleste] ne laisse pas beaucoup de place au doute :

Ce n'est que chez mon psychothérapeute que j'ai découvert la vérité sur la question de savoir pourquoi les Tchèques me plaisent tant. Quand j'écris des récits et des reportages sur l'histoire de la Tchécoslovaquie, sur des personnages que je considère avec surprise (tous ces types à la fois kafkaïens et orwelliens), tout le temps, j'écris sur moi ${ }^{62}$.

Tel est donc le sens enfin dévoilé du projet tchèque de Mariusz Szczygieł : la psychothérapie personnelle d'un écrivain polonais athée, mal à l'aise dans sa culture d'origine et terrifié par la maladie et la mort ${ }^{63}$. La volonté d'être la (mauvaise) conscience de la Pologne, comme Thomas Bernhard était celle de l'Autriche ${ }^{64}$, s'efface en fin de compte devant le malaise intime: Szczygieł s'est forgé l'image accueillante d'une culture tchèque aux antipodes de la culture polonaise pour y chercher refuge et consolation. La communauté secrète des "Tchécophiles» et des "crypto-Tchèques» polonais, dont Mariusz Szczygieł se voulait le porte-parole, doit se sentir trahie quand elle lit sous sa plume : «toute ma prétendue tchécophilie n'était que le détour que je faisais vers la

mariusz-gottland> [consulté le 26/08/2015) : il est flatteur pour les Tchèques, dit-il, de se sentir aimés par un étranger; c'est surtout la raison pour laquelle ils aiment ce livre.

6I. Voir <http://video.aktualne.cz/dvtv/spisovatel-szczygiel-cesi-nejsou-poseroutkove/ r -3c8a44de27aemie480e50025 90ofeaO4/> [consulté le 26/08/2015].

62. M. Szczygieł, Láska nebeská [Amour céleste], ouvr. cité, $4^{\mathrm{e}}$ de couverture de la version tchèque : "Teprve u svého psychoterapeuta jsem objevil pravdu - proč se mi Čechy tak líbi. Když pišu príběhy a reportáže o historii Československa, o postavách, nad kterými vrtím hlavou - takových kafkovsko-orwellovských typech-, pišu celou dobu o sobè."

63. À l'image du nain mélancolique qui orne la couverture tchèque de Láska nebeská [Amour céleste], où il est permis de voir la figuration de la personnalité de l'auteur. La photo est de F. Dostál.

64. M. Szczygieł, Chacun son paradis, ouvr. cit, p. 34 (version française). 
polonité6s». Certes, on ne peut que se réjouir de cette réconciliation avec soimême au spectacle de laquelle nous sommes conviés, même s'il ne s'agit que d'une rechute provisoire de polonité. Mais qu'en est-il des Tchèques? Il me semble que c'est eux qui s'en sortent le plus mal, car ils ont été d'une certaine façon instrumentalisés : le seul reproche véritable qu'on puisse faire à Mariusz Szczygieł, c'est en effet d'avoir au passage confortéles stéréotypes qui composent le mythe national tchèque, à des fins à la fois idéologiques et personnelles, au lieu de soumettre ce mythe à l'examen critique dont il a tant besoin de la part de la littérature.

65. M. Szczygiet, Láska nebeská [Amour céleste], ouvr. cité, p. I68 (version tchèque) : «[...] celé to moje predstíráni čechofila bylo mou objižd'kou k polskosti» (trad. M.-O. Thirouin). 